\title{
A European party with all-American technology: The case of DiEM25.
}

\author{
Camille Akmut
}

August 24, 2019

\begin{abstract}
Raw research on a European party's use of technologies. Results : 0\% European - based on their own information. Recommendations are given. An exemplary case, only.
\end{abstract}




\section{Data}

Table 1: DiEM25 technologies

\begin{tabular}{|c|c|c|c|c|}
\hline Type & Product & Company & Location & \\
\hline content & Google Maps & Google & U.S.A. & \\
\hline content & YouTube & Google & U.S.A. & \\
\hline typography & Google Fonts & Google & U.S.A. & \\
\hline anti-spam & Google reCAPTCHA & Google & U.S.A. & \\
\hline payment & & Stripe & U.S.A. & \\
\hline payment & & PayPal & U.S.A. & \\
\hline hosting & Heroku & U.S.A. & \\
\hline mass mailing & Mailchimp & & U.S.A. & \\
\hline mass mailing & SparkPost & & U.S.A. & \\
\hline autom. phone calls/texts & Twilio & & U.S.A. & \\
\hline various (reverse proxy) & & Cloudflare & U.S.A. & \\
\hline \hline & & & E.U. & $0 \%$ \\
\hline & & & U.S.A. & $100 \%$ \\
\hline
\end{tabular}

\section{Recommendations}

In a recent address to the Greek Parliament, Yanis Varoufakis lectured the Minister of Digital Policy on proper uses of technology : not Google products, but creations by national companies should be used. There is nothing to oppose to this : he should start at home, in his own party.

DiEM25 ought to be a role model for all other European parties, and not lagging behind as its does where socially responsible uses of technology are concerned.

It should be praised however for its transparency, which made this study possible. But, transparency is only the first step : transformation next. (Varoufakis will recall a certain German philosopher's theses...)

And, last but not least, even though this study has concentrated on geographical location, hence jurisdiction, it should be noted that this is only one criterion : whether free software is used is just as important; most listed above are not, they are what is called proprietary.

\begin{tabular}{|c|c|c|}
\hline Policy topic & Priority & Recommendation \\
\hline Hosting & Highest & Move your hosting to a E.U. country. \\
\hline Payment & Medium & Pioneer alternative payment methods. \\
\hline Google Maps & Medium & Use e.g. OpenStreetMap instead. \\
\hline Google Fonts & Low & This is completely unnecessary. Use other fonts. \\
\hline
\end{tabular}


By law, a European party should only be allowed to have their servers in the E.U..

Your data and your members' have no place on a server somewhere in San Francisco... (Did this occur to no one?)

\section{Conclusion}

"The only technology we need is democracy", a technological adviser for this party has boldly and unrepentantly advanced. We feel much sympathy for such acts of bravado, but they are not enough.

As long as we are dependent on them, we must learn the technologies that surround us - or it is them that dominate us;

"in ways that escape us", we wrote, in what feels centuries ago... 


\section{References}

https://www.iubenda.com/privacy-policy/49614846 (policy summary) https://www.iubenda.com/privacy-policy/49614846/legal (full policy)

("Latest update: April 07, 2019")

Marx, Karl. Theses on Feuerbach (in The German Ideology).

Varoufakis, Yanis. 2019. "Yanis Varoufakis' speech on the first day of the new term of the Greek Parliament - DiEM25". /watch?v=T8nEjW8i394 